\title{
Pneumocystis carinii pneumonia: a light microscopical and ultrastructural study
}

\author{
PS HASLETON, A CURRY, EM RANKIN \\ From the Department of Pathology, Withington Hospital, and the Department of Medical Oncology, \\ Christie Hospital, Manchester
}

SUMMARY A case of Pneumocystis carinii pneumonia developing in a patient with treated diffuse $\frac{\Omega}{8}$ lymphocytic lymphoma is described. The electron microscopic features and life cycle of the organism? are illustrated. The patient died twenty-four hours after the biopsy had been taken. Septrin appeared $\stackrel{\omega}{\oplus}$ to have affected the trophozoite stage. Pneumocystis carinii appears to induce interstitial pulmonary $\vec{O}$ oedema and fibrosis. A discussion of the role of electron microscopy in early diagnosis of the disease is presented.

Pneumocystis carinii pneumonia occurs in debilitated infants but is also found in patients with cancer who are receiving chemotherapy. The most common clinical situation where this type of infection occurs is in patients with haematological malignancies receiving combination chemotherapy. As combination chemotherapy becomes more widely used for other malignancies it is possible that the incidence of the disease will increase. Relatively few reports of the electron microscopy of $\boldsymbol{P}$ carinii have been made. ${ }^{1-5}$ Electron microscopy is useful in diagnosis, especially if a rapid technique is used. It also helps to explain some of the underlying pathological changes that are not obvious on light microscopy. We present a case report of $\boldsymbol{P}$ carinii pneumonia with ultrastructural studies and a comment on the effect of treatment on the course of the disease.

\section{Case report}

The patient was a caucasian male aged 18 who presented with a six week history of an enlarging lymph node in the right submandibular region in December 1978. He was referred to the Christie Hospital, Manchester. The biopsy was classified as a poorly differentiated diffuse lymphocytic lymphoma (Rappaport classification). There was no history of weight loss, fevers or night sweats. There was no evidence of lymphoma deposits in the marrow aspirate though a clot section showed a moderate excess of lymphocytes. The white blood count was $4600 / \mu 1\left(4.6 \times 10^{9} / 1\right)$ with $43 \%$ lymphocytes. An

Accepted for publication 6 April 1981 enlarged node in the left axilla indicated clinical stage II A disease.

He was treated with radiotherapy to the head and $\mathscr{\Phi}^{\circ}$ neck followed by chemotherapy with vincristine, $\overrightarrow{\vec{\theta}}$ adriamycin and prednisolone followed 18 6-mercaptopurine, methotrexate and cyclophofphamide as oral maintenance. He remained well fo 12 months but then developed weight loss wi anorexia. In January 1980 a chest radiograph showed upper zone fibrosis consistent with the previous radiotherapy. A month later he was unwell with $\stackrel{\mathbb{Q}}{\stackrel{2}{ }}$ persistent cough, morning sputum and sweats. $\mathrm{He} \overrightarrow{\vec{B}}$ did not respond to ampicillin and was admitted to 3 hospital. The patient was ambulent on arrival at $כ$ hospital but went into respiratory failure on the fourth day after admission despite treatment with broad spectrum antibiotics including intravenous? Septrin. A chest radiograph showed marked deterioration with widespread opacity in all lung zones. $A \stackrel{-}{\circ}$ percutaneous lung biopsy was carried out as he was judged too ill to withstand an open lung biopsy. $\mathrm{He}$ developed a pneumothorax and a chest drain was $\subseteq$ inserted. After the biopsy he received $100 \%$ oxygen $I$ but died on 22 February 1980, 24 hours later.

\section{Material and methods}

The lung biopsy consisted of a cylinder of grey $\mathrm{\omega}$ tissue. Pieces were taken from each end and fixed in $3 \%$ cacodylate-buffered glutaraldehyde. Some of these pieces were processed rapidly $(3 \mathrm{~h})$ for electron $\mathbb{\Phi}$ microscopy, ${ }^{6}$ other pieces were processed more ${ }^{+}$ conventionally for electron microscopy. ${ }^{?}$ The $\frac{0}{0}$ material was embedded in Epon resin; semithin $\underset{\mathbb{D}}{\stackrel{O}{*}}$ 1138 
$(1 \mu \mathrm{m})$ sections were cut and stained with toluidine blue for light microscope examination prior to ultrathin sections being cut from the same blocks. Ultrathin sections were cut on either an LKB IV or Reichert OMU4 ultramicrotome, stained with uranyl acetate and lead citrate and examined in an AEI (Kratos) EM 801 electron microscope.

The main mass of lung tissue was processed conventionally in paraffin wax and $5 \mu \mathrm{m}$ sections were cut. Haematoxylin and eosin as well as methanamine silver stains were done on the paraffin sections. Necropsy tissues were processed in paraffin wax and stained as above.

\section{Results}

Light microscopy showed two main features. The first was an interstitial plasma cell infiltrate. Secondly, some alveoli were filled with an exudate that had a honeycomb appearance (Fig. 1). On staining with methanamine silver there were organisms lying in the alveolar spaces (Fig. 2). These were $4 \mu \mathrm{m}$ in diameter, circular and had several dense foci in the middle of the organism. There was no evidence of cytomegalovirus infection. A patchy early interstitial pulmonary fibrosis was present.

Electron microscopy showed different forms of Pneumocystis in the alveolar lumen (Fig. 3). There were two basic forms, the trophozoite and the cyst, although gradations between these forms were present.

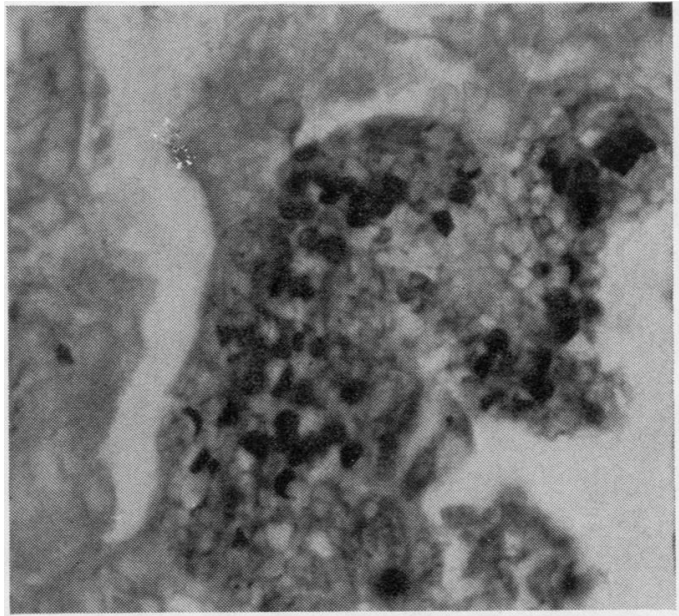

Fig. 2 Pneumocystis carinii stained by methanamine silver $\times 640$

\section{TROPHOZOITE}

These highly pleomorphic forms ranged in length from 2 to $12 \mu \mathrm{m}$. Some had a fairly smooth outline (Fig. 4) whereas others had fine tubular surface projections $0.1 \mu \mathrm{m}$ in diameter (Fig. 5). The plasma membrane was invariably covered externally by an electron dense layer about $40 \mathrm{~nm}$ thick. Internally organelles often appeared poorly preserved but some contained an easily recognisable nucleus bounded by a double membrane and sometimes showed a per-

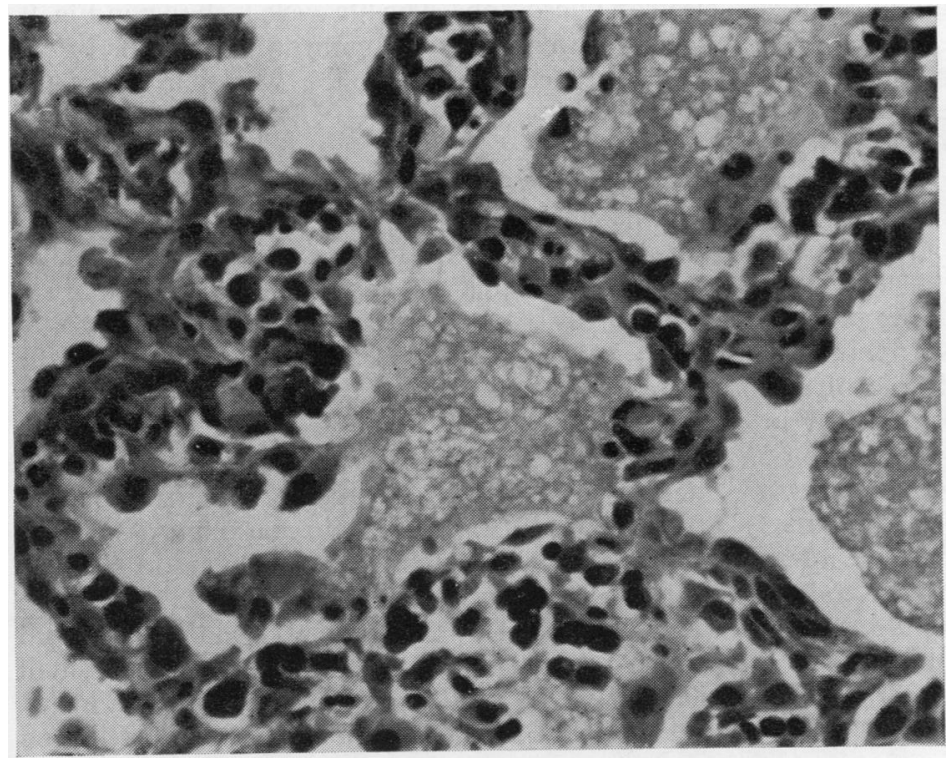

Fig. 1 Foamy exudate in alveolar lumina and plasma cell infiltrate in alveolar wall. Haematoxylin and eosin $\times 322$ 


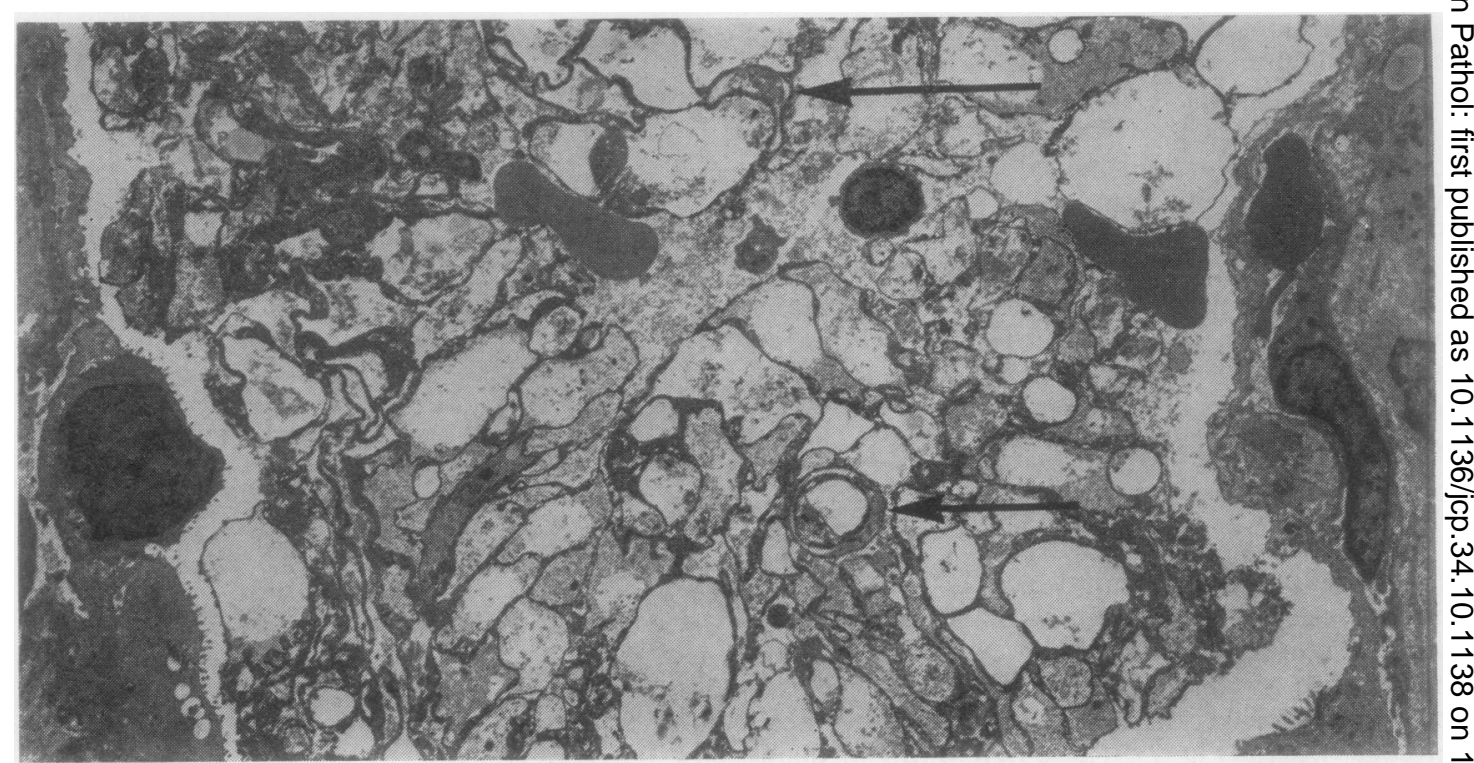

Fig. 3 Low power electron micrograph showing cysts (arrowed) in centre of alveolar lumen and trophozoites. Uranyl acetate and lead citrate $\times 2620$

ipheral electron dense condensation which possibly represented a nucleolus. Some endoplasmic reticulum and a small number of mitochondria were also present. The granular cytoplasmic matrix contained medium electron dense vacuoles, $400 \mathrm{~nm}$ in diameter, reminiscent of lipid; $65 \mathrm{~nm}$ electron dense granules and microtubules. Glycogen, in rosette form, was present in some cells. Some larger trophozoites often appeared "empty" or contained large electron lucent areas.

Occasionally, trophozoites with transverse walls were seen (Fig. 6), possibly representing binary fission. Rarely, trophozoites were found between alveolar cells indicating invasion of the alveolar wall (Fig. 7).

\section{CYST}

Four forms of the cyst were recognised, the precyst, the mature cyst, the cyst liberating its trophozoites and finally, the empty collapsed cyst devoid of trophozoites (Fig. 8). The cyst was almost circular in outline, 3.5 to $4 \mu \mathrm{m}$ in diameter and was devoid of surface projections. The wall surrounding the cyst was more complex than that of the trophozoite, being composed of a unit membrane, an intermediate $25-40 \mathrm{~nm}$ thick electron lucent layer and an external $25 \mathrm{~nm}$ thick electron dense layer. The cytoplasm of the cyst showed a gradation of complexity. The least complex forms (precysts) showed no internal compartmentation and contained the organelles previously described within the txos phozoite cytoplasm. More complex forms (matte cysts) contained intracystic bodies which appearedo identical in structure in trophozoites. Other cysts appeared to show the release of these small tro phozoites through a gap in the cyst wall. Collapse of the empty cyst produced the empty crescent-shaped which was the most easily identifiable form in the electron microscope. The life cycle is shown in Fig. 9. The cysts were mainly present in the middle of the alveoli whereas trophozoites were close to the alveolar walls.

HOST RESPONSE TO PNEUMOCYSTIS
Oedema fluid, erythrocytes and occasional poly morphonuclear leucocytes were found in the alveolaro lumen. Some leucocytes contained vacuoles which enclosed easily recognisable remains of trophozoites and cysts (Fig. 8). Plasma cells were not seen in thes alveolar lumina and only infrequently in the alveolar? wall. Some type I and type II pneumocytes showed ballooning of their cytoplasm. There was prominene interstitial pulmonary oedema associated with 0 fibrosis (Fig. 10). There was no marked proliferation $\mathrm{W}_{\mathrm{W}}$ of type II cells or prominence of intra-alveolaro macrophages. At necropsy (Dr KV Lodge) there was enlargement of the cervical, abdominal, porta and tracheobronchial lymph nodes. There were smalis bilateral pleural effusions. The lungs were heavyo (right, $1630 \mathrm{~g}$, left, $1570 \mathrm{~g}$ ) and firm with a mottled redō 


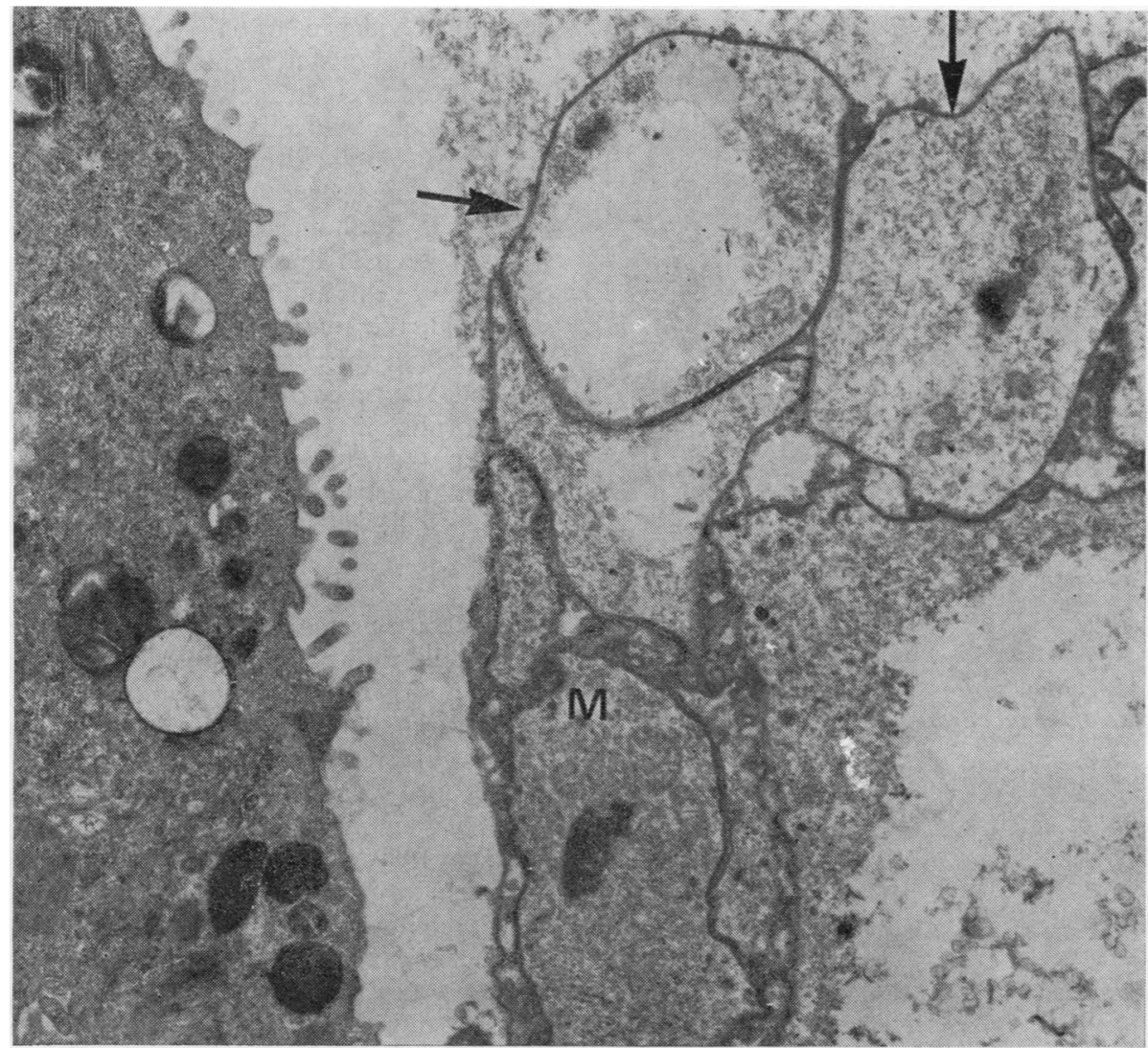

Fig. 4 Predominantly smooth outlined trophozoites (arrows) with part of a type II pneumocyte showing microvilli. Mitochondria $(M)$ are present in one trophozoite. Uranyl acetate and lead citrate $\times 16000$

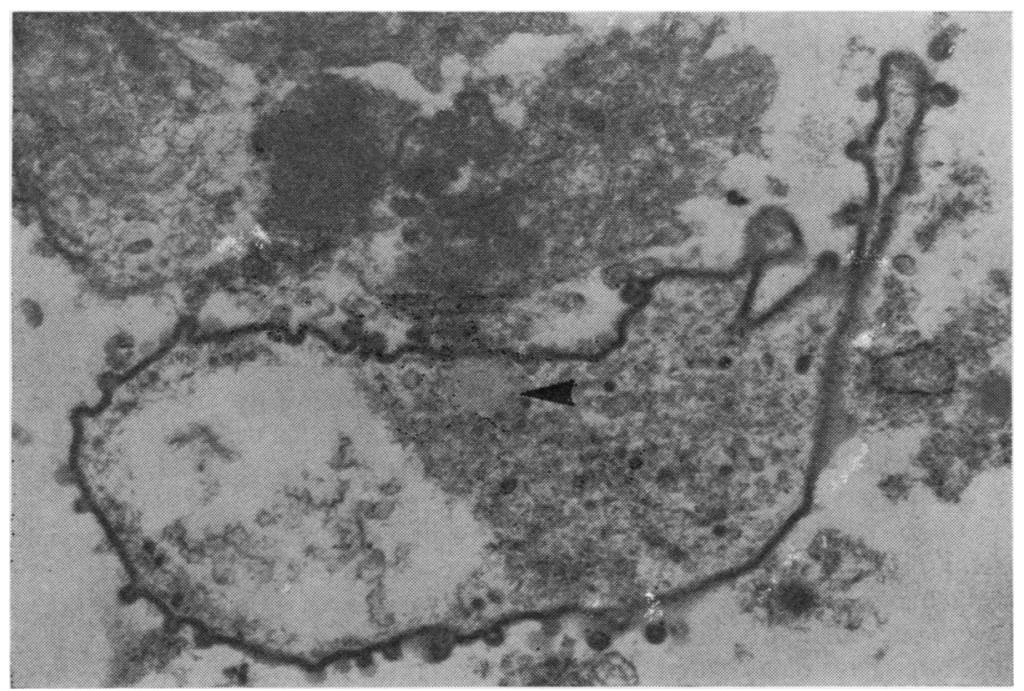

Fig. 5 Trophozoite with surface projections confluent with cell membrane. The cytoplasm contains electron dense granules and a medium electron dense deposit reminiscent of lipid (arrow). Uranyl acetate and lead citrate $\times 13500$ 


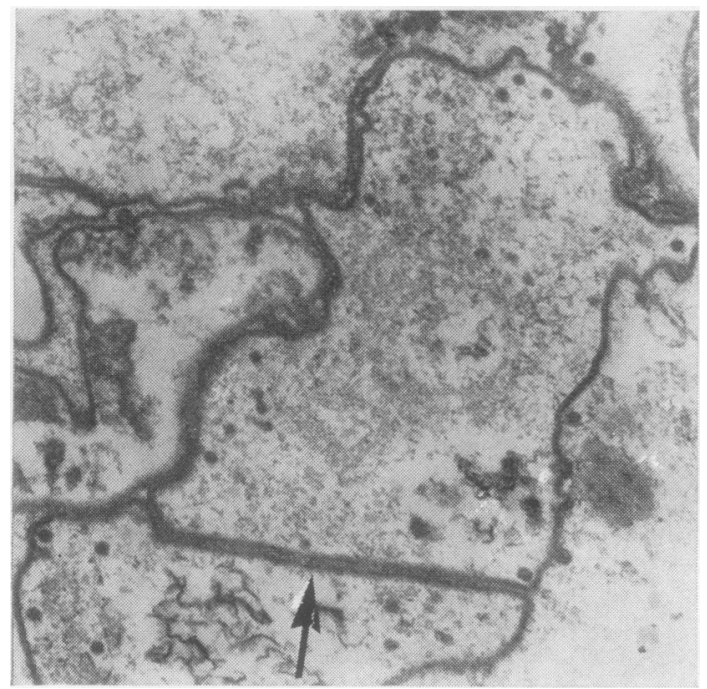

Fig. 6 Trophozoite with transverse wall (arrow). Uranyl acetate and lead citrate $\times 22000$

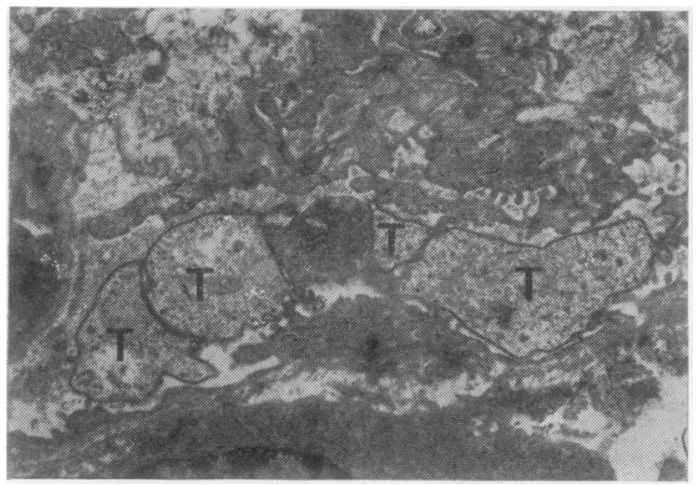

Fig. 7 Trophozoites $(T)$ in alveolar wall along with part of a plasma cell. Uranyl acetate and lead citrate $\times 7500$

and white surface. On the cut surface the lung was solid with many firm white areas measuring up to $1 \mathrm{~cm}$ in diameter. The bronchi contained mucus and some pus. The liver $(1630 \mathrm{~g})$ and spleen $(330 \mathrm{~g})$ were enlarged.

Histologically, the lymph nodes showed diffuse lymphocytic lymphoma and the pulmonary pathology had progressed from the description above. There was interstitial fibrosis and also marked interstitial oedema (Fig. 11). A cuboidal epithelium lined the alveoli. The honeycomb type exudate persisted and hyaline membranes were present lining many alveoli. Some alveoli showed intra-alveolar oedema. Occasional giant endothelial cells were present and microthrombi were seen in some pulmonary arterioles denoting evidence of disseminated intravascularo coagulation. Focal hyperplasia of muscle was seen in the walls of alveolar ducts. Plasma cells were present:in the alveolar wall and there were foci of neutrophi polymorphs in the alveolar lumina and alveolar wall Methanamine silver staining for $\boldsymbol{P}$ carinii was positive in the honeycomb areas and the organism $\bar{x}$. was also seen in the lumina of pulmonary arteries, pulmonary veins and capillaries. No organisms were seen inside the alveolar lining cells or in the alveolares wall on light microscopy. A fibrinous pleurisy was $\overrightarrow{0}$ present. The myocardium showed foci of neutrophitpolymorphs in the interstitium and interstitia oedema. No $P$ carinii was identified in the myo cardium, liver, spleen or kidney by light microscopy

\section{Discussion}

Clinically the present case showed a terminal $P$ carini $\overrightarrow{\vec{w}_{0}}$ pneumonia in a patient undergoing treatment for $a_{\infty}^{\infty}$ diffuse poorly differentiated lymphocytic lymphoma? In the early stages of the illness the symptoms were non-specific, namely, weight loss and anorexia, ando indeed until six weeks before death, the patient hado been playing squash. The chest radiograph in January 1980 showed only upper lobe fibrosis consistent with the previous radiotherapy buto ${ }^{\circ}$ month later there were prominent radiological sig with a widespread diffuse alveolar pattern affect俉星 all lung zones. In previous clinical reviews, ${ }^{8-10}$ stress is rightly put on the pulmonary signs and symptoms with malaise, cough, tachypnoea and fever. Physica signs may be minimal though cyanosis and rales are most frequently reported. Though his weight loss and $\vec{b}$ anorexia may be related to the underlying lym 3 phoma, it is equally likely that they are related to the presence of $\boldsymbol{P}$ carinii infection. Hughes ${ }^{10}$ noted weight. loss in infants with the disease but does not stateon when this occurs.

The light microscopic features of the organism will not be discussed here as they are adequately dealt with in standard texts. It should be added however, that histological diagnosis, even witho methanamine silver stains, is not straightforward Structures resembling $\boldsymbol{P}$ carinii cysts were seen in theo lungs of alloxan diabetic rabbits. ${ }^{11}$ These were shown to be fungal spores and the authors recommended ao direct fluorescent antibody test to distinguish then two organisms. $P$ carinii also has to be distinguishedn from erythrocytes but this can be achieved with $a_{0}^{\omega}$
good silver stain.

The rapid embedding technique, which can yielde electron microscopic results in 3-4 hours, ${ }^{6}$ may be of use in the rapid diagnosis of pneumocystis pneumonia. It is obvious that the organism is easilyo identified by electron microscopy. However, becauseâ 

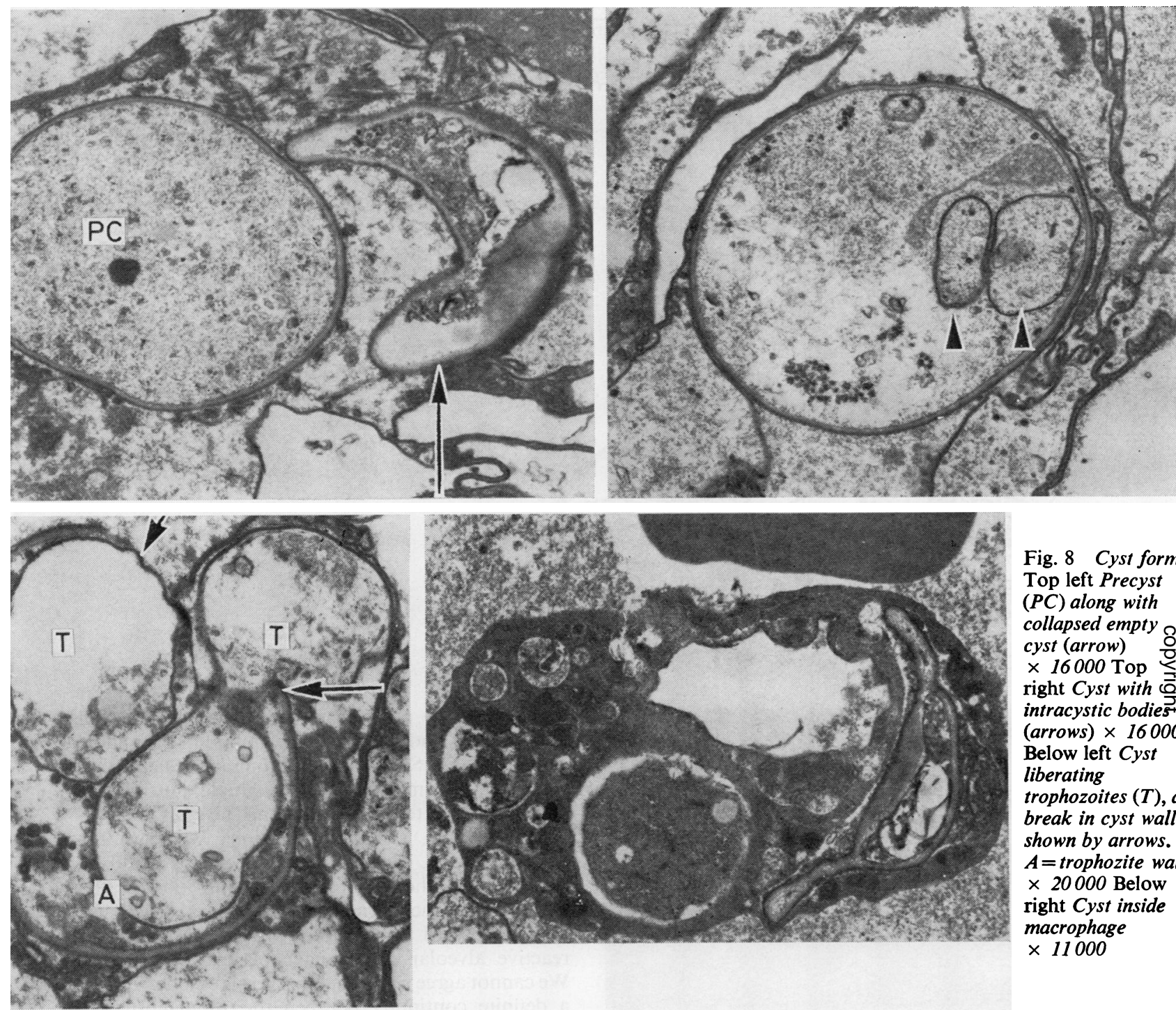

Fig. 8 Cyst forms

Top left Precyst (PC) along with collapsed empty cyst (arrow) $\times 16000$ Top right Cyst with 흥 intracystic bodiest (arrows) $\times 16000$ 응 Below left Cyst liberating trophozoites $(T), a \overrightarrow{\vec{B}}$ break in cyst wall $\frac{0}{3}$ shown by arrows. $A=$ trophozite wall $\times 20000$ Below right Cyst inside macrophage $\times 11000$

electron microscopy utilises such small samples, careful selection of the site of biopsy and also of the pieces used for rapid embedding must be made. In addition, semithin, fairly large area sections, stained with toluidine blue and examined by light microscopy can show areas most likely to yield positive results. These areas can be trimmed and ultrathin sections cut prior to electron microscopic examination. However, in our somewhat limited experience, the resin used in the rapid embedding technique can sometimes fail to penetrate the specimen adequately and thus the relatively long conventional processing is a useful safeguard.
Electron microscopy can be used to give a definite diagnosis, as in the present case. Several different stages of the organism were recognised. The trophozoite had a pleomorphic outline and was sometimes covered with surface projections. In most of the trophozoites there were few organelles though some showed endoplasmic reticulum, nuclei and a few mitochondria. It is of interest that some trophozoites showed transverse walls suggestive of binary fission.

The trophozoites formed cysts and the most easily recognisable was the collapsed cyst which had a crescentic shape and stained positively with meth- 


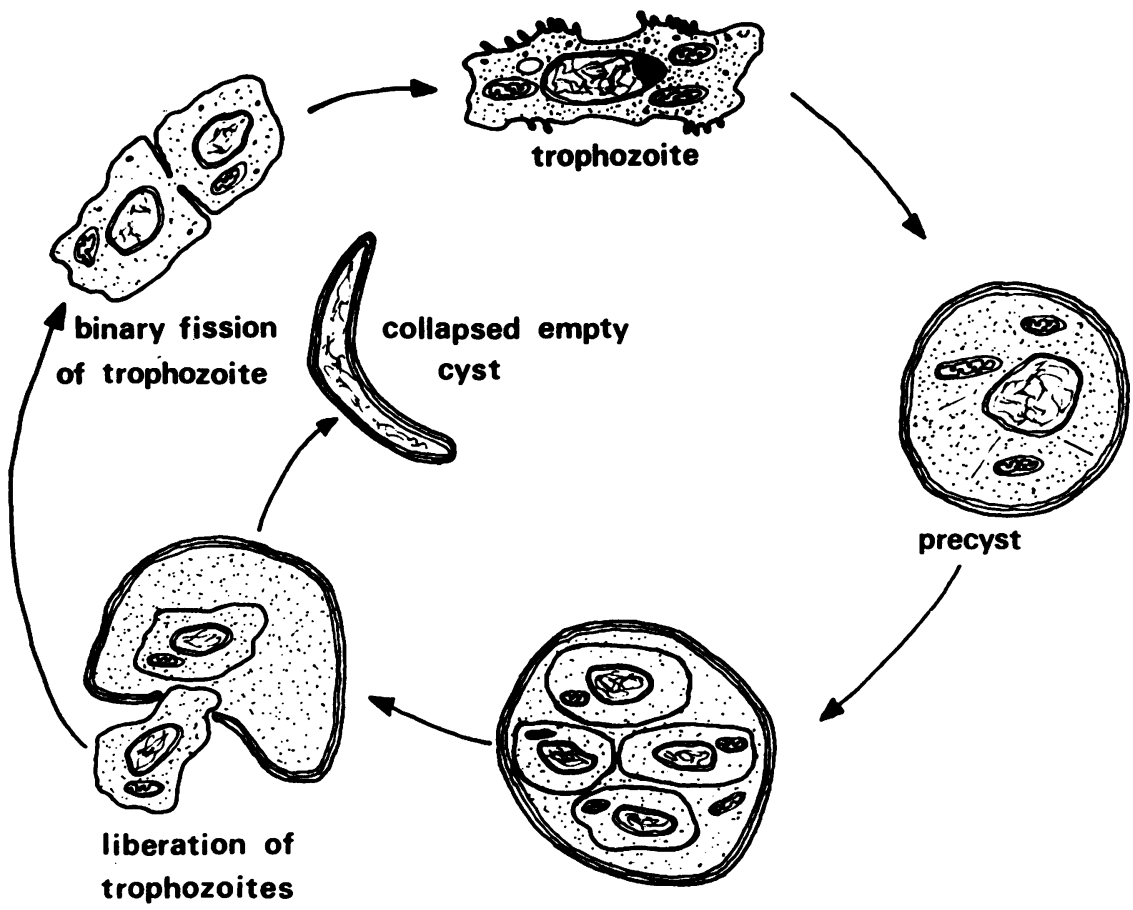

Fig. 9 Life cycle of Pneumocystis carinii

cyst with intracystic bodies

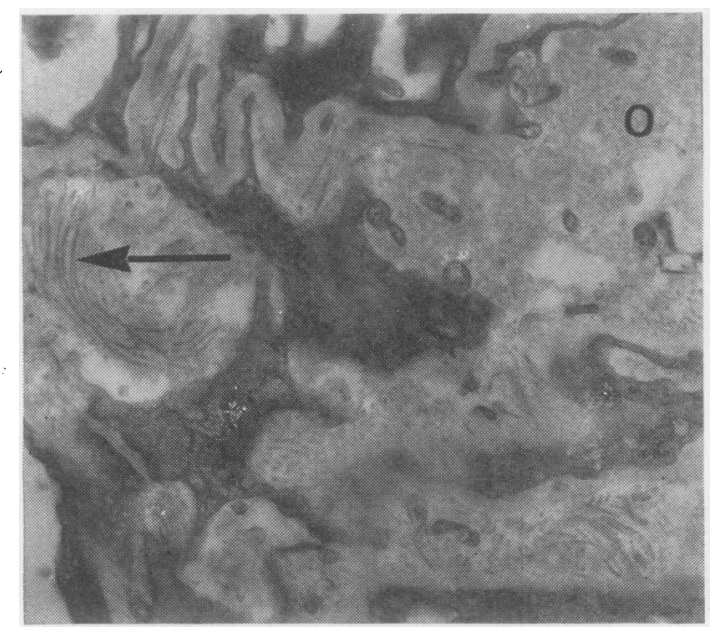

Fig. 10 Alveolar wall with oedema $(O)$ and collagen fibres (arrow). Uranyl acetate and lead citrate $\times 13500$

anamine silver. The trophozoites also stained positively with methanamine silver, but because of their thinner wall, did not stain as intensely.

In this case there was a tendency in the sections examined by electron microscopy for the tro- phozoites to be present nearest to the alveolar wall and the cysts and precysts to be present in the middle of the alveolus. Such a distribution may indicate that limitation of some factor-for example, nutrition or oxygen, stimulates cyst formation. The cysts and precysts have no surface projections whereas trophozoites have. These surface projections could be important in the maintenance of nutrition of the organism by increasing absorptive area. Price and Hughes ${ }^{4}$ regard these surface projections as part of reactive alveolar macrophage plasma membranes. We cannot agree with this for several reasons. Firstly, a definite continuity between the tubular surface projections and the trophozoites could be observed. Secondly, in the biopsy specimen macrophages were infrequent but such surface projections were numerous. Finally, in the experimental animal given chlorphentermine, an appetite suppressant which causes histiocyte proliferation in the lungs, no similar material was seen. ${ }^{12}$ Vavre and Kucera ${ }^{3}$ also noted that such surface projections were an integral part of the parasite. Mobility or food engulfment are unlikely uses of the surface projections since no contractile elements could be seen. The pleomorphic form of the organism does, however, suggest that the organism may be capable of amoeboid movement. 


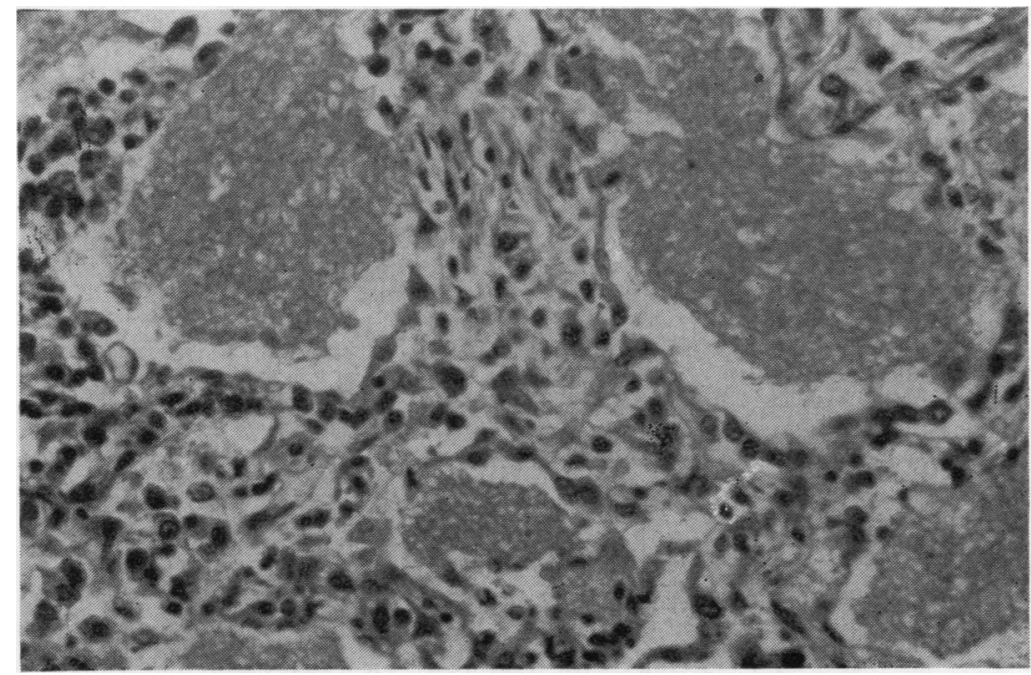

Fig. 11 Lung at post mortem showing increased thickness of alveolar wall as well as foamy exudate in alveolar lumina $\times 450$

Some authors have regarded these tubular expansions of membranes as myxoviruses. ${ }^{13}$ In rats it is thought that the tubular expansions increase the surface area of the organism and anchor the parasite to the alveolar epithelium. ${ }^{3}$ However, in the present case there was a clear space between organism and alveolar epithelium but it is impossible to say if this is due to shrinkage during processing for electron microscopy.

$\boldsymbol{P}$ carinii is known to be sensitive to both pentamidine and Septrin.10 A comparison can be made between the ultrastructural characteristics in our case, who had had intravenous Septrin for 24 hours and animal studies ${ }^{313}$ where no therapy had been given. Assuming good preservation in our case, the main target appears to be the trophozoite. In our case some trophozoites often appeared empty or contained large electron lucent areas whilst other trophozoites showed little internal structure. Campbell ${ }^{2}$ compared the electron microscopic findings in one case with and one without pentamidine treatment. He noted ghost trophozoites and also failed to find mature cysts with intracystic bodies in his treated case.

Finally, comment must be made on the pulmonary fibrosis and the interstitial oedema which may have preceded it. Many previous papers have neglected the alveolar wall and its response to $\boldsymbol{P}$ carinii. The present case is complicated in that the patient had received radiotherapy as well as chemotherapy with drugs including cyclophosphamide, both of which can induce interstitial pulmonary fibrosis. Even though there are many causes for such fibrosis, the advancing nature of the fibroblastic reaction raises the possibility of $\boldsymbol{P}$ carinii causing or potentiating the problem. Other authors have noted pulmonary fibrosis after pentamidine therapy ${ }^{44-16}$ but this drug was not used and Septrin is not known to cause pulmonary fibrosis. The only other factor that may be important is the administration of oxygen. In Sevitt's series ${ }^{17}$ the earliest a patient developed diffuse oxygen pneumonitis was after $2 \frac{1}{2}$ days of $80-100 \%$ oxygen and in this case with minor proliferation $( \pm)$. We consider it probably too early for oxygen to have caused its damage as the oxygen was only given for 24 hours. There was clear evidence of disseminated intravascular coagulation in the present case as shown by giant endothelial cells and intravascular fibrin thrombi.

The alveolar cells appear to react fairly minimally to the organism. There is a mild proliferation of type II pneumocytes on electron microscopy but there was no marked desquamation of these cells such as is seen in busulphan lung. ${ }^{18}$ Type I pneumocytes showed occasional vacuolation of their cytoplasm but were otherwise normal. There was no marked increase in alveolar macrophages.

We wish to thank Professor Derek Crowther and Dr K Carroll who provided clinical details, Dr KV Lodge who made available the postmortem report and histological sections and Mrs Cheryl Anfield for her technical assistance.

\section{References}

${ }^{1}$ Huneycutt HC, Anderson WR, Hendry WS. Pneumocystis carinii pneumonia. Case studies with electron microscopy. Am J Clin Pathol 1964;41:411-8.

2 Campbell WG. Ultrastructure of pneumocystis in human lung. Life cycle in human pneumocystosis. Arch Pathol 
$1972 ; 93: 312-24$

${ }^{3}$ Vavre J, Kucera K. Pneumocystis carinii Delanoë, its ultrastructure and ultrastructural affinities. $J$ Protozool 1970;17:463-83.

${ }^{4}$ Price RA, Hughes WT. Histopathology of Pneumocystis carinii infestation and infection in malignant disease in childhood. Hum Pathol 1974;5:737-52.

${ }^{5}$ Sueishi K, Hisano S, Sumiyoshi A, Tanaka K. Scanning and transmission electron microscopic study of human pulmonary pneumocystosis. Chest 1977;72:213-6.

${ }^{6}$ Rowden G, Lewis MG. Experience with a three-hour electron microscopy biopsy service. J Clin Pathol 1974; 27:505-10.

7 Harris M. Differential diagnosis of spindle cell tumours by electron microscopy - personal experience and a review. Histopathology $1981 ; 5: 81-105$.

${ }^{8}$ Walzer PD, Perl DP, Krogstad DJ, Rawson PG, Shultz MG. Pneumocystis carinii pneumonia in the United States: Epidemiologic, diagnostic and clinical features. Ann Intern Med 1974;80:83-93.

${ }^{9}$ DeVita VT, Goodell B, Hubbard S, Geelhoed GW, Young RC. Pneumocystis pneumonia in patients with cancer: clinical setting. Natl Cancer Inst Monogr 1976; 43:41-6.

${ }^{10}$ Hughes WT. Pneumocystis carinii pneumonia. $N$ Engl $J$ Med 1977;297:1381-3.

${ }^{11}$ Reinhardt DJ, Kaplan W, Chandler FW. Morphologic resemblance of zygomete spores to Pneumocystis carinii cysts in tissue. Am Rev Respir Dis 1977;115:170-1.

${ }^{12}$ Heath D, Smith P, Hasleton PS. Effects of chlorphentermine on the rat lung. Thorax 1973;28:551-8.

${ }^{13}$ Tamura T, Ueda K, Furuta T, Goto Y, Fujiwara K. Electron microscopy of spontaneous pneumocystosis in a nude mouse. Jpn J Exp Med 1978;48:363-8.

${ }^{14}$ Burke BA, Goode RA. Pneumocystis carinii infection. Medicine (Baltimore) 1973;52:23-51.

${ }^{15}$ Dutz W, Post C, Vissal K, Kohout E. Endemic infantile Pneumocystis carinii infection: the Shiraz study. Natl Cancer Inst Monogr 1976;43:31-8.

${ }^{16}$ Young RC, DeVita VT. Treatment of Pneumocystis carinii pneumonia: current status of the regimens of pentamidine isethionate and pyrimethamine-sulfadiazine. Natl Cancer Inst Monogr 1976;43:193-8.

${ }^{17}$ Sevitt S. Diffuse and focal oxygen pneumonitis. A preliminary report on the threshold of pulmonary oxygen toxicity in man. J Clin Pathol 1974;27:21-34.

${ }^{18}$ Littler WA, Kay M, Hasleton PS, Heath D. Busulphan lung. Thorax 1969;24:639-55.

Requests for reprints to: Dr PS Hasleton, Department of Pathology, Withington Hospital, West Didsbury, Manchester M20 8LR, England. 